\title{
Forced convection and sedimentation past a flat plate
}

\author{
By NIKOLAOS A. PELEKASIS AND ANDREAS ACRIVOS \\ The Levich Institute, City College of The City College of the City University of New York. \\ 140th St \& Convent Ave, New York NY, 10031, USA
}

(Received 8 August 1994 and in revised form 5 January 1995)

The steady laminar flow of a well-mixed suspension of monodisperse solid spheres, convected steadily past a horizontal flat plate and sedimenting under the action of gravity, is examined. It is shown that, in the limit as $R e \rightarrow \infty$ and $\epsilon>0$, where $R e$ is the bulk Reynolds number and $\epsilon$ is the ratio of the particle radius $a$ to the characteristic length scale $L$, the analysis for determining the particle concentration profile has several aspects in common with that of obtaining the temperature profile in forcedconvection heat transfer from a wall to a fluid stream moving at high Reynolds and Prandtl numbers. Specifically, it is found that the particle concentration remains uniform throughout the $O\left(R e^{-1 / 2}\right)$ thick Blasius boundary layer except for two $O\left(e^{2 / 3}\right)$ thin regions on either side of the plate, where the concentration profile becomes nonuniform owing to the presence of shear-induced particle diffusion which balances the particle flux due to convection and sedimentation. The system of equations within this concentration boundary layer admits a similarity solution near the leading edge of the plate, according to which the particle concentration along the top surface of the plate increases from its value in the free stream by an amount proportional to $X^{5 / 6}$, with $X$ measuring the distance along the plate, and decreases in a similar fashion along the underside. But, unlike the case of gravity settling on an inclined plate in the absence of a bulk flow at infinity considered earlier (Nir \& Acrivos 1990), here the concentration profile remains continuous everywhere. For values of $X$ beyond the region near the leading edge, the particle concentration profile is obtained through the numerical solution of the relevant equations. It is found that, as predicted from the similarity solution, there exists a value of $X$ at which the particle concentration along the top side of the plate attains its maximum value $\phi_{m}$ and that, beyond this point, a stagnant sediment layer will form that grows steadily in time. This critical value of $X$ is computed as a function of $\phi_{s}$, the particle volume fraction in the free stream. In contrast, but again in conformity with the similarity solution, for values of $X$ sufficiently far removed from the leading edge along the underside of the plate, a particle-free region is predicted to form adjacent to the plate. This model, with minor modifications, can be used to describe particle migration in other shear flows, as, for example, in the case of crossflow microfiltration.

\section{Introduction}

We consider the steady laminar flow of a suspension of sedimenting monodisperse solid particles past a horizontal flat plate. Far from the plate, the free-stream velocity $U_{\infty}$ and particle concentration $\phi_{s}$ are both uniform. We also suppose that the particles are small enough for their Reynolds number, based on their diameter and either the 
Stokes sedimentation velocity or the shear rate inside the viscous layer formed near the plate, to be very small.

This flow situation constitutes a fairly general prototype for studying the effect of shear-induced particle migration in shear layers. It is also a natural extension of the problem considered by Nir \& Acrivos (1990) in their study of sedimentation onto an inclined plate, in which a concentrated sediment flows along the inclined plate owing to gravity. In that case, the shear created within the flowing sediment gives rise to shear-induced particle diffusion which opposes the sedimentation flux and prevents the particles from accumulating onto the upper surface of the plate. This occurs within a thin region close to the wall, termed the viscous layer, where viscous forces are balanced by buoyancy. In fact, the structure of that solution has several points in common with that of free-convection heat transfer from a vertical plate at high Grashof and Prandtl numbers with the notable difference that the particle concentration profile is discontinuous across the interface separating the suspension from the flowing concentrated sediment. Nir \& Acrivos (1990) derived expressions for the particle velocity and concentration profiles within this sediment layer on the basis of an effective continuum model, valid for very small particle sizes relative to the characteristic length scale of the flow, which incorporated the effects of shear-induced particle diffusion (Leighton \& Acrivos 1986). Their model was recently extended by Kapoor \& Acrivos (1995) who also tested experimentally a number of theoretical predictions pertaining to the thickness of the sediment layer and the corresponding particle velocity profiles, and confirmed the theoretical results which were arrived at through $a b$ initio calculations that did not entail the use of adjustable parameters.

In view of the reliability of the effective medium model for buoyancy-driven flow, it would be of interest to consider an analogous situation in which forced convection plays an important role. In the problem examined here particles sediment onto the plate, where they tend to accumulate forming a concentrated sediment. At the same time, owing to the interaction between the free stream and the plate, a Blasius-type thin shear layer is formed near the plate, which subsequently induces shear-induced particle resuspension and thereby reduces their degree of accumulation. In addition, this whole process is affected by the presence of convective particle transport. In fact, as will be seen, an estimate of the size of the region within which the particle volume fraction variation is confined can be obtained simply by balancing the corresponding convective and diffusive particle fluxes.

The present study constitutes a first attempt towards constructing a more comprehensive model of particle migration in shear layers which could then be used to describe various processes of significant practical interest. Such an example is crossflow microfiltration, in which a suspension of neutrally buoyant rigid spherical particles is made to flow in a channel having a flat porous bottom surface, under conditions where a pressure drop is imposed between the suspension and the permeate side of the microporous membrane. Consequently, fluid is sucked through the membrane, whereas the particles accumulate along the surface, forming a thin concentrated particle layer. Moreover, if this suction velocity is small relative to that in the centre of the channel, then, for all practical purposes, the flow within the bulk of the suspension is fully developed and is not affected significantly by particle migration. As a result, a known tangential shear stress is exerted along the particle layer which, together with convection, will determine the concentration profile (Davis \& Leighton 1987). In a different context, the shear-induced diffusion of raindrops as they approach an airfoil can affect the structure of the thin water film on its surface whose existence may be responsible for the observed loss in efficiency of airfoils under heavy rain and 
high wind shear (Dunham 1987). Detailed analysis of these two flow situations is left for a future investigation; however, the mathematical and physical similarities between them and the problem at hand are fairly evident.

In $\S 2$ the problem is formulated mathematically on the basis of the effective-medium model, while in $\S 3$ the basic equations are reduced to their boundary-layer form. It will be seen that the structure of the mathematical system is similar to that which describes laminar heat transfer from a flat plate to a fluid stream moving past it at high Reynolds and Prandtl numbers. In $\S 4$, a similarity solution is constructed valid near the leading edge of the plate according to which the particle concentration within the diffusion layer approaches smoothly its constant value, $\phi_{s}$, in the adjacent momentum layer without experiencing the discontinuity found by Nir \& Acrivos (1990) in the absence of forced convection. Finally, in $\$ 5$, the results of a numerical solution to the boundary-layer equations are presented which extend the theory beyond the leading edge of the plate. It will be seen that the particle concentration along the top side of the plate increases monotonically from its value at the tip, $\phi_{s}$, until it reaches its maximum possible value, $\phi_{m}$, at which point a stagnant layer is formed the thickness of which will increase continuously in time. In contrast, along the underside of the plate, the particle concentration decreases monotonically until a particle-free region is attained.

\section{Problem formulation}

We wish to examine the flow of a suspension of non-colloidal spherical solid particles in a viscous fluid convected steadily towards a semi-infinite flat plate, as shown in figure 1 , and sedimenting under the action of gravity. The particles are supposed to be small enough that their inertia is negligible. In the following $a$ will denote the particle radius, $U_{\infty}$ the free-stream velocity, and $\rho_{s}$, and $\mu_{s}$, respectively, the effective density and viscosity of the suspension in the free stream, where the particle volume fraction $\phi$ equals $\phi_{s}$. The suspension will be modelled as an effective continuum Newtonian fluid with concentration-dependent physical properties.

In this context the volumetric average velocity of the binary mixture is defined as

$$
\boldsymbol{u}=\boldsymbol{u}_{p} \phi+\boldsymbol{u}_{f}(1-\phi)
$$

where $\boldsymbol{u}_{p}, \boldsymbol{u}_{f}$ are the particle and fluid ensemble-averaged velocities at any location in the mixture. Owing to the incompressibility of the fluid and of the solid particles, the effective suspension density becomes

$$
\rho(\phi)=\rho_{f}+\left(\rho_{p}-\rho_{f}\right) \phi \equiv \rho_{s} \gamma(\phi)
$$

where $\rho_{f}$ and $\rho_{p}$ are the pure fluid and solid particle densities respectively and $\gamma(\phi)$ is the effective density of the mixture rendered dimensionless with $\rho_{s}$. Similarly, the effective viscosity $\mu(\phi)$ is set equal to $\mu_{s} \lambda(\phi)$. The equations governing the motion of the mixture are rendered dimensionless using $L$, as yet unspecified, as the characteristic length scale, $U_{\infty} \equiv\left|\boldsymbol{U}_{\infty}\right|$ as the characteristic velocity, and the inertial scale $\rho_{s} U_{\infty}^{2}$ for the pressure. Consequently, following Nir \& Acrivos (1990) the continuity and momentum equations for the mixture become, respectively,

$$
\boldsymbol{\nabla} \cdot \boldsymbol{u}=0
$$

and $\quad \operatorname{Re} \gamma(\phi) u \cdot \nabla u=-\operatorname{Re} \nabla P+\nabla \cdot\left[\lambda(\phi)\left(\nabla u+\nabla \boldsymbol{u}^{T}\right)\right]+\frac{g}{g} \frac{9}{2} \frac{G r}{\operatorname{Re}}\left(\phi-\phi_{s}\right)$,

where $\boldsymbol{g}$ is the gravitational acceleration. Besides $\phi_{s}$ the dimensionless parameters characterizing the flow are: the Reynolds number, $R e=U_{\infty} \rho_{s} L / \mu_{s}$, the Grashof 


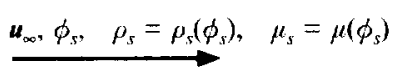

$\downarrow g$

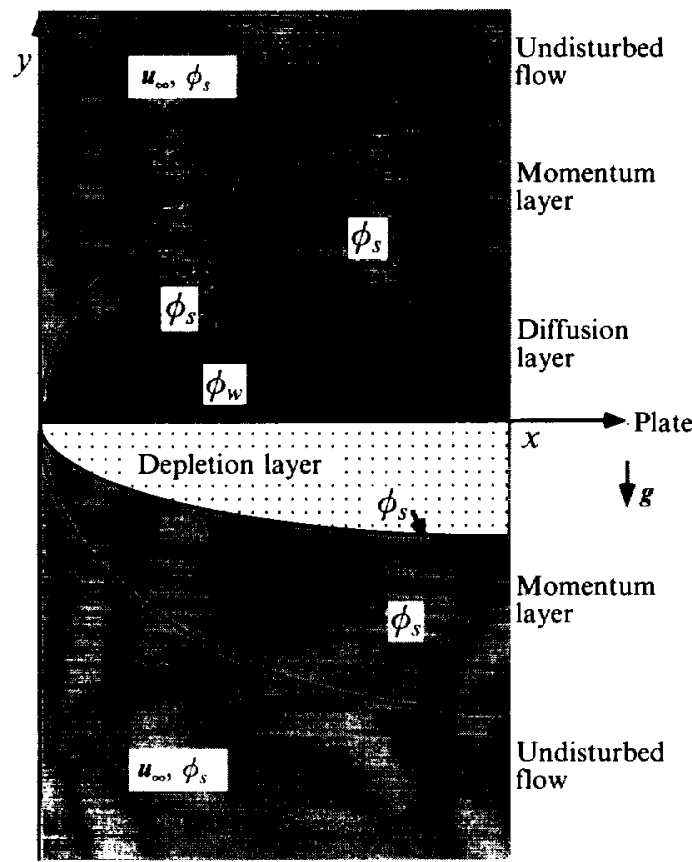

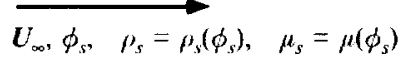

Figure 1. Schematic of the flow.

number, $G r=\frac{2}{9}\left(\rho_{p}-\rho_{f}\right) g \rho_{s} L^{3} / \mu_{s}^{2}$, and the size ratio $\epsilon=a / L ; \epsilon \ll 1$ for the effectivemedium model to be valid. The hydrostatic pressure, $\left(\rho_{p}-\rho_{f}\right) \phi_{s} g y$, has been incorporated into the pressure field $P$, which now vanishes far from the plate (henceforth, $x$ and $y$ will indicate, respectively, the Cartesian coordinates along and perpendicular to the plate as shown in figure 1).

In dimensional form, the total particle flux is given by

$$
J=-D_{1} \nabla \phi-\frac{D_{2}}{\mu(\phi)|\nabla u|} \nabla(\mu(\phi)|\nabla u|)+\left(u^{*}+u\right) \phi,
$$

with

$$
D_{1}=|\nabla u| a^{2} \beta(\phi), \quad D_{2}=|\nabla u| a^{2} K_{c} \phi^{2},
$$

where $|\nabla \boldsymbol{u}| \equiv\{\boldsymbol{\nabla} \boldsymbol{u}: \boldsymbol{\nabla} \boldsymbol{u}\}^{1 / 2}$ is the norm of the local gradient of the bulk velocity field (Leighton \& Acrivos, $1987 a, b$ ), $\boldsymbol{u}^{*}$ is the particle slip velocity,

$$
\boldsymbol{u}^{*}=\boldsymbol{u}_{p}-\boldsymbol{u}=u_{t} f(\phi) \frac{g}{g}, \quad u_{t}=\frac{2}{9} \frac{g a^{2}\left(\rho_{p}-\rho_{f}\right)}{\mu_{f}}, \quad f(\phi)=\frac{1-\phi}{\lambda(\phi)},
$$

and $\mu_{f}$ is the pure fluid viscosity. The first two terms on the right-hand side in (2.5) account for the diffusive flux of the particles due to concentration and shear rate gradients, respectively (Leighton \& Acrivos 1987a,b), whereas the last term corresponds to the convective flux. In addition, the particle slip velocity, given by the 
first equality in (2.7), is set equal to the Stokes terminal velocity $u_{t}$ multiplied by a hindrance function $f(\phi)$ which accounts for the influence of particle interactions. The expression for $f(\phi)$ shown in (2.7) is that used by Nir \& Acrivos (1990). The expressions given above for $D_{1}, D_{2}$ and $\boldsymbol{u}^{*}$ presuppose that the particle Reynolds number, whether in terms of the sedimentation velocity or the local bulk shear rate, is vanishingly small.

In terms of the dimensionless variables introduced earlier, the steady-state particle balance equation becomes

$$
\boldsymbol{u} \cdot \boldsymbol{\nabla} \phi=\epsilon^{2} \nabla \cdot\left[\beta(\phi)|\nabla u| \nabla \phi+\frac{K_{c} \phi^{2}}{\lambda(\phi)} \nabla(\lambda(\phi)|\nabla u|)-\frac{g}{g} \frac{G r}{R e} \phi f(\phi)\right] .
$$

At the solid boundary, the no-slip condition is applied together with the requirement that the total particle flux at the plate be equal to zero, i.e.

$$
\boldsymbol{u}=0 \quad \text { and }\left[\beta(\phi)|\nabla u| \nabla \phi+\frac{K_{c} \phi^{2}}{\lambda(\phi)} \nabla(\lambda(\phi)|\nabla u|)-\frac{\boldsymbol{g}}{g} \frac{G r}{R e} \phi f(\phi)\right] \cdot \boldsymbol{n}=\mathbf{0},
$$

where $\boldsymbol{n}$ is the unit vector normal to the surface. At large distances from the wall the flow is uniform and the suspension is well mixed, hence

$$
\boldsymbol{u}=\boldsymbol{U}_{\alpha}, \quad \phi=\phi_{s} .
$$

In performing the calculations to be reported below, the relative effective viscosity of the mixture was taken to be of the form

$$
\lambda(\phi)=\frac{\mu(\phi)}{\mu_{s}}=\frac{\left(1+1.5 \phi / 1-\phi / \phi_{m}\right)^{2}}{\left(1+1.5 \phi_{s} / 1-\phi_{s} / \phi_{m}\right)^{2}}, \quad \phi_{m}=0.58
$$

(Leighton \& Acrivos $1987 b$ ), where $\phi_{m}$ is the maximum possible particle volume fraction in the flowing suspension. Also, following Leighton \& Acrivos (1987b), we let

$$
\beta(\phi)=\frac{1}{3} \phi^{2}\left(1+\frac{1}{2} \mathrm{e}^{8.8 \phi}\right) \text { and } K_{c}=0.6 \text {. }
$$

\section{Boundary-layer structure when $R e \gg 1$ and $\epsilon \ll 1$}

We consider the case of uniform flow past a flat plate at zero angle of attack. For large values of $R e$, based on the free-stream velocity $U_{x}$ and the length scale $L$, an $O\left(R e^{-1 / 2}\right)$ vorticity layer is formed near the plate. Above this region, the flow is uniform and the suspension is well mixed. Applying the standard Blasius-type boundary-layer scaling transformations

$$
U=u, \quad V=v R e^{1 / 2}, \quad X=x, \quad Y=y R e^{1 / 2},
$$

with $u$ and $v$ denoting the longitudinal and transverse velocity components, respectively, we obtain, for the continuity, momentum and mass transfer equations,

$$
\begin{gathered}
\frac{\partial U}{\partial X}+\frac{\partial V}{\partial Y}=0 \\
\gamma(\phi)\left(U \frac{\partial U}{\partial X}+V \frac{\partial U}{\partial Y}\right)=\frac{\partial}{\partial Y}\left[\lambda(\phi) \frac{\partial U}{\partial Y}\right]-\frac{\partial P}{\partial X} \\
\frac{\partial P}{\partial Y}=\frac{9}{2} \frac{G r\left(\phi-\phi_{s}\right)}{R e^{5 / 2}} \\
U \frac{\partial \phi}{\partial X}+V \frac{\partial \phi}{\partial Y}=\epsilon^{2} \operatorname{Re}^{3 / 2} \frac{\partial}{\partial Y}\left[\left|\frac{\partial U}{\partial Y}\right| \beta(\phi) \frac{\partial \phi}{\partial Y}+\frac{K_{c} \phi^{2}}{\lambda(\phi)} \frac{\partial}{\partial Y}\left(\lambda(\phi)\left|\frac{\partial U}{\partial Y}\right|\right)\right]+\frac{\epsilon^{2} G r}{\operatorname{Re}^{1 / 2}} \frac{\partial}{\partial Y}[\phi f(\phi)]
\end{gathered}
$$


as well as the zero-flux condition at the plate,

$$
Y=0, \quad \beta(\phi)\left|\frac{\partial U}{\partial Y}\right| \frac{\partial \phi}{\partial Y}+\frac{K_{c} \phi^{2}}{\lambda(\phi)} \frac{\partial}{\partial Y}\left(\lambda(\phi)\left|\frac{\partial U}{\partial Y}\right|\right)+\phi f(\phi) \frac{G r}{R e^{2}}=0 .
$$

Next, we examine the magnitude of $\epsilon^{2} R e^{3 / 2}$ which can be rearranged into

$$
\epsilon^{2} R e^{3 / 2}=\left(\frac{a}{L}\right)^{2}\left(\frac{U_{\infty} L \rho_{s}}{\mu_{s}}\right)^{3 / 2}=\frac{U_{\infty}^{3 / 2}}{L^{1 / 2} v_{s}^{1 / 2}} \frac{a^{2}}{\nu_{s}}=\left[\frac{U_{\infty}\left(L U_{\infty} / v_{s}\right)^{1 / 2}}{L}\right] \frac{a^{2}}{\nu_{s}}
$$

where $v_{s}=\mu_{s} / \rho_{s}$. Now, the term in square brackets is a measure of the shear rate near the plate as a result of the existence of a Blasius-type boundary layer, hence the entire expression corresponds to the shear particle Reynolds number within the boundary layer. As mentioned in the previous section, this was already assumed to be a small number in the context of our analysis. Having thus established that $\epsilon^{2} R e^{3 / 2}$ is very small whenever the particle Reynolds number is sufficiently small, we can proceed with the analysis in a fashion similar to the case of heat transfer to a fluid stream convected past a flat plate at high $R e$ and $P r$.

As $\epsilon^{2} R e^{3 / 2} \rightarrow 0$, particle diffusion, both below and above the plate, is confined within a narrow layer attached to the plate which is embedded within the momentum layer as $Y \rightarrow 0$. But, from the Blasius solution of the momentum boundary-layer equations, we know that, as $Y \rightarrow 0, U \sim Y, V \sim Y^{2}$ (Schlichting 1968), and therefore, by requiring that the diffusive terms in (3.5) balance those due to convection, we conclude that the thickness of the diffusion layer must be $O\left(e^{2 / 3} R e^{1 / 2}\right)$. This in turn leads to the transformations

$$
\tilde{Y}=\frac{Y}{\epsilon^{2 / 3} R e^{1 / 2}}, \quad \tilde{U}=\frac{U}{\epsilon^{2 / 3} R e^{1 / 2}}, \quad \tilde{V}=\frac{V}{\left(\epsilon^{2 / 3} R e^{1 / 2}\right)^{2}},
$$

so that within the diffusion layer we obtain, respectively, for the momentum and continuity equations

and

$$
\begin{gathered}
\frac{\partial}{\partial \tilde{Y}}\left[\lambda(\phi) \frac{\partial \tilde{U}}{\partial \tilde{Y}}\right]=O\left(\epsilon^{2} R e^{3 / 2}\right), \\
\frac{\partial P}{\partial \tilde{Y}}=O\left(\epsilon^{2 / 3} \frac{G r}{R e^{2}}\right) \\
\frac{\partial \tilde{U}}{\partial X}+\frac{\partial \tilde{V}}{\partial \tilde{Y}}=0
\end{gathered}
$$

In the next section we will see that, in the context of our analysis, $\epsilon^{2 / 3} G r / R e^{2} \rightarrow 0$, which results in a uniform pressure distribution. Further, in view of (3.9), the shear stress remains constant across the diffusion layer and hence shear-induced particle diffusion takes place only because of gradients in the particle concentration. In terms then of the new coordinates, the particle balance equation becomes

$$
\tilde{U} \frac{\partial \phi}{\partial X}+\tilde{V} \frac{\partial \phi}{\partial \tilde{Y}}=\frac{\partial}{\partial \tilde{Y}}\left[\beta(\phi)\left|\frac{\partial \tilde{U}}{\partial \tilde{Y}}\right| \frac{\partial \phi}{\partial \tilde{Y}}\right]+\frac{\epsilon^{2 / 3} G r}{R e^{3 / 2}} \frac{\partial}{\partial \tilde{Y}}[\phi f(\phi)]
$$

with boundary conditions

$$
\begin{gathered}
\tilde{Y}=0, \quad \beta(\phi)\left|\frac{\partial \tilde{U}}{\partial \tilde{Y}}\right| \frac{\partial \phi}{\partial \tilde{Y}}+\phi f(\phi) \frac{\epsilon^{2 / 3} G r}{R^{3 / 2}}=0, \\
\tilde{Y} \rightarrow \pm \infty, \quad \phi \rightarrow \phi_{s}
\end{gathered}
$$


where the negative $\tilde{Y}$-values denote the region underneath the plate. We note that, in contrast to the conventional cases involving mass transfer across boundary layers, here the diffusion coefficient, which appears both in the particle balance equation (3.12) as well as in the boundary condition (3.14), is proportional to the local shear rate rather than being constant. This, as we shall see presently, affects the solution of (3.12) in a significant way.

\section{Similarity solution valid near the leading edge of the plate}

As $\epsilon^{2 / 3} \mathrm{Gr} / \operatorname{Re}^{3 / 2} \rightarrow 0$ particle transfer due to sedimentation vanishes so that $\phi \rightarrow \phi_{s}$ and the Blasius velocity profile applies throughout the boundary layer. Therefore, we construct the leading-edge expansion

$$
\phi=\phi_{s}+\frac{\epsilon^{2 / 3} G r}{\operatorname{Re}^{3 / 2}} \Psi(X, \tilde{Y})+\ldots
$$

which, when substituted into (3.9)-(3.12), yields, to $O\left(\epsilon^{2 / 3} G r / R e^{3 / 2}\right)$,

$$
\left.\begin{array}{c}
\frac{\partial^{2} \tilde{U}}{\partial \tilde{Y}^{2}}=0, \\
\frac{\partial \tilde{U}}{\partial X}+\frac{\partial \tilde{V}}{\partial \tilde{Y}}=0, \\
\tilde{U} \frac{\partial \Psi}{\partial X}+\tilde{V} \frac{\partial \Psi}{\partial \tilde{Y}}=\beta\left(\phi_{s}\right) \frac{\partial}{\partial \tilde{Y}}\left[\left|\frac{\partial \tilde{U}}{\partial \tilde{Y}}\right| \frac{\partial \Psi}{\partial \tilde{Y}}\right], \\
\tilde{Y}=0, \quad \beta\left(\phi_{s}\right)\left|\frac{\partial \tilde{U}}{\partial \tilde{Y}}\right| \frac{\partial \Psi}{\partial \tilde{Y}}+\phi_{s} f\left(\phi_{s}\right)=0, \\
\tilde{Y} \rightarrow \pm \infty, \quad \Psi \rightarrow 0 .
\end{array}\right\}
$$

We see then that, to this order of approximation, the shear rate within the diffusion layer, $\partial \tilde{U} / \partial \tilde{Y}$, is everywhere equal to $\pm 0.332 X^{-1 / 2}$, as given, for $Y \rightarrow 0$, by the Blasius solution for the corresponding constant-property case. In addition, the term containing the settling rate only appears in the boundary condition at the wall. It is this constant term in (4.5) that accounts for the increase in the particle concentration within the diffusion layer, or for the particle depletion below the plate.

Letting, then,

$$
\tilde{U}= \pm \frac{0.332}{X^{1 / 2}} \tilde{Y} \text { and } \quad \tilde{V}= \pm \frac{0.332 \tilde{Y}^{2}}{4 X^{3 / 2}}, \quad \tilde{Y} \gtrless 0
$$

in (4.2)-(4.5), we reduce the problem to a PDE whose form along with its boundary conditions suggests the existence of a similarity solution. Indeed, by introducing the transformations

$$
\eta=\frac{\tilde{Y}}{\left[\beta\left(\phi_{s}\right)\right]^{1 / 3} X^{1 / 3}}, \quad \Psi=\frac{\phi_{s} f\left(\phi_{s}\right) X^{5 / 6} F_{1}(\eta)}{0.332\left[\beta\left(\phi_{s}\right)\right]^{2 / 3}},
$$

we obtain the ODE

$$
\pm \frac{5}{6} \eta F_{1}(\eta) \mp \frac{\eta^{2}}{12} \frac{\mathrm{d} F_{1}}{\mathrm{~d} \eta}=\frac{\mathrm{d}^{2} F_{1}}{\mathrm{~d} \eta^{2}}, \quad \eta \gtrless 0
$$




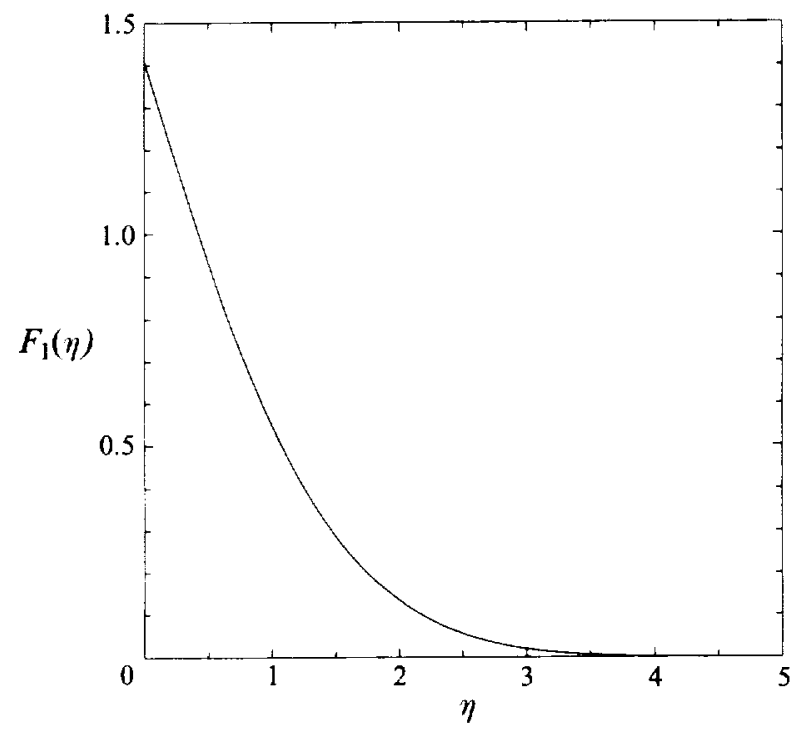

FIgURE 2. Similarity solution obtained by solving (4.8) for positive $\eta$.

with boundary conditions

$$
\begin{gathered}
\frac{\mathrm{d} F_{1}}{\mathrm{~d} \eta}=-1 \quad \text { as } \quad \eta \rightarrow \pm 0, \\
F_{1} \rightarrow 0 \quad \text { as } \quad \eta \rightarrow \pm \infty
\end{gathered}
$$

which can readily be solved numerically to give $F_{1}(\eta)$, shown in figure 2 . In addition it can easily be seen that $F_{1}(\eta)=-F_{1}(-\eta)$ with a discontinuity at $\eta=0$. Also $F_{1}\left(0^{+}\right)=$ 1.41 .

To a first approximation then, within the diffusion layer, whose thickness relative to the length scale $L$ is $O\left(R e^{-1 / 2} \epsilon^{2 / 3} R e^{1 / 2}\right)=O\left(\epsilon^{2 / 3}\right)$, the particle concentration is given by

$$
\phi=\phi_{s}+F_{1}(\eta) \frac{X^{5 / 6} \phi_{s} f\left(\phi_{s}\right)}{0.332\left[\beta\left(\phi_{s}\right)\right]^{2 / 3}} \frac{\epsilon^{2 / 3} G r}{R e^{3 / 2}}, \quad \eta \gtrless 0,
$$

implying that, along the top face of the plate, the increase in $\phi$ from its value in the free stream is proportional to $X^{5 / 8}$, whereas, below the plate, $\phi$ decreases in a similar fashion. It should also be noted that, unlike the similarity solution given by Nir \& Acrivos (1990) for sedimentation over an inclined plate, here the particle concentration profile inside the diffusion layer, both above and below the plate, approaches continuously $\phi_{s}$, its value in the undisturbed flow. In fact, an asymptotic analysis of (4.8) reveals that, as $\eta \rightarrow \pm \infty, F_{1}(\eta) \sim \mathrm{e}^{\mp \eta^{3} / 36}$. The reason for this difference in the two concentration profiles, is that, in our case, the shear rate remains constant at any given $X$ throughout the diffusion layer, whereas in Nir \& Acrivos (1990) the corresponding shear rate is a monotonically decreasing function of $Y$ and vanishes at infinity.

Up to this point, the characteristic length scale $L$ used in our analysis has been left unspecified. In what follows it proves convenient, however, to choose $L$ such that

$$
\frac{\epsilon^{2 / 3} G r}{\operatorname{Re}^{3 / 2}} \frac{\phi_{s} f\left(\phi_{s}\right)}{0.332\left[\beta\left(\phi_{s}\right)\right]^{2 / 3}}=1,
$$


giving $\quad L \equiv a\left[\frac{0.332\left[\beta\left(\phi_{s}\right)\right]^{2 / 3}}{\phi_{s} f\left(\phi_{s}\right)}\left(\frac{a U_{\infty}}{v_{s}}\right)^{3 / 2} \frac{v_{s}^{2} \rho_{s}}{a^{3} g \Delta \rho}\right]^{6 / 5}, \Delta \rho \equiv \rho_{p}-\rho_{f}$

in which case (4.11) becomes

$$
\phi=\phi_{s}+F_{1}(\eta) X^{5 / 6}+\ldots \quad \text { as } \quad X \rightarrow 0 .
$$

We note that if $L$ is chosen according to (4.13),

$$
\epsilon^{2} \operatorname{Re}^{3 / 2} \sim\left(\frac{a^{3} g \Delta \rho}{v_{s}^{2} \rho_{s}}\right)^{3 / 5}\left(\frac{U_{\infty} a}{v_{s}}\right)^{3 / 5}
$$

which, for small particles, is normally very small, as required by our analysis. In the context of crossflow microfiltration Davis \& Sherwood (1990) obtained a similarity solution that has some of the characteristics of the one presented here. For instance, in their analysis, the thickness of the sheared concentration boundary layer is also proportional to $X^{1 / 3}$. Of course, their study is different in that they neglect gravity settling, they consider fully developed flow in the bulk of the suspension as opposed to the Blasius-type vorticity layer considered here, and finally they focus on that portion of the membrane where the particles form a thin stagnant sediment layer, cake, with concentration $\phi_{m}=0.58$. However, if one focuses on the entrance portion of the membrane where the flow is not fully developed and the cake layer has not yet formed, $\phi_{w}<\phi_{m}$, and provided the transmembrane pressure drop is much larger than the down-channel pressure drop, in which case the wall permeation velocity, $v_{w}$, is taken to be constant, it can be seen that, as $X \rightarrow 0$, a similarity solution exists which is exactly the same as the one presented here with $L$ defined such that

$$
\frac{\sigma}{\epsilon^{4 / 3} \operatorname{Re}^{1 / 2}} \frac{\phi_{s}}{0.332\left[\beta\left(\phi_{s}\right)\right]^{2 / 3}}=1
$$

and

$$
\sigma=\frac{v_{w}}{U_{x}} \ll R e^{-1 / 2}, \quad \epsilon^{2} R e^{3 / 2} \ll 1 .
$$

Substituting in (4.16) the parameter values used by Davis \& Birdsell (1987), $\phi_{s}=0.02, U_{x}=3.7 \mathrm{~cm} \mathrm{~s}^{-1}, v_{w}=3.0 \times 10^{-3} \mathrm{~cm} \mathrm{~s}^{-1}, a=2 \times 10^{-2} \mathrm{~cm}, \mu=20 \mathrm{cP}, \rho=$ $1.18 \mathrm{~g} \mathrm{~cm}^{-3}$, in their experimental investigation, we obtain $L \approx 2 \mathrm{~cm}$, which conforms to the conditions in (4.17). When $X \sim L$ the similarity solution is not valid and the full equations have to be solved; they are only slightly different from the full equations derived in the next section to provide the location $X_{c r}$ for which the stagnant cake layer forms. This is a very important design parameter as it controls the plugging of the channel.

\section{Solution valid when $X \sim O(1)$}

Introducing the further transformation

$$
\bar{Y}=\tilde{Y} /\left[\beta\left(\phi_{s}\right)\right]^{1 / 3}
$$

we obtain for the momentum equation (3.9)

$$
\frac{\partial}{\partial \bar{Y}}\left[\lambda(\phi) \frac{\partial \tilde{U}}{\partial \bar{Y}}\right]=0
$$


which, when combined with the asymptotic form of the Blasius solution as $Y \rightarrow 0$ given by (4.6), leads to

$$
\tilde{U}= \pm\left[\beta\left(\phi_{s}\right)\right]^{1 / 3} \frac{0.332}{X^{1 / 2}} \int_{0}^{\bar{Y}} \frac{\mathrm{d} t}{\lambda(\phi)}
$$

and

$$
\tilde{V}= \pm\left[\beta\left(\phi_{s}\right)\right]^{2 / 3} \frac{0.332}{2 X^{3 / 2}} \int_{0}^{\bar{Y}}\left\{\frac{(\bar{Y}-t)}{\lambda(\phi)}\left[1+\frac{2 X(\mathrm{~d} \lambda / \mathrm{d} \phi)(\partial \phi / \partial X)}{\lambda(\phi)}\right]\right\} \mathrm{d} t
$$

on account of the continuity equation. Substituting the above into (3.11)-(3.13), we then obtain for the particle concentration equation

$$
\begin{aligned}
\pm \frac{1}{X^{1 / 2}}\left[\int_{0}^{\bar{Y}} \frac{\mathrm{d} t}{\lambda(\phi)}\right] \frac{\partial \phi}{\partial X} \pm \frac{1}{2 X^{3 / 2}}\left\{\int_{0}^{\bar{Y}} \mathrm{~d} t \frac{(\bar{Y}-t)}{\lambda(\phi)}[1\right. & \left.\left.+\frac{2 X(\mathrm{~d} \lambda / \mathrm{d} \phi)(\partial \phi / \partial X)}{\lambda(\phi)}\right]\right\} \frac{\partial \phi}{\partial \bar{Y}} \\
& =\frac{\partial}{\partial \bar{Y}}\left[\frac{\Delta(\phi)}{X^{1 / 2}} \frac{\partial \phi}{\partial \bar{Y}}+F(\phi)\right], \quad \bar{Y} \gtrless 0,
\end{aligned}
$$

valid for all $X$, subject to the boundary conditions

$$
\begin{gathered}
\frac{\partial \phi}{\partial \bar{Y}}+X^{1 / 2} \frac{F(\phi)}{\Delta(\phi)}=0 \quad \text { at } \quad \bar{Y}=0, \\
\phi \rightarrow \phi_{s} \quad \text { as } \quad \bar{Y} \rightarrow \pm \infty, \quad \phi \rightarrow \phi_{s} \quad \text { as } \quad X \rightarrow 0, \\
F(\phi)=\frac{\phi f(\phi)}{\phi_{s} f\left(\phi_{s}\right)}, \quad \Delta(\phi)=\frac{\beta(\phi)}{\beta\left(\phi_{s}\right) \lambda(\phi)} .
\end{gathered}
$$

with

As $X \rightarrow 0$ the similarity solution can easily be recovered. It is also not difficult to show that the first correction to the similarity solution will be of the form $F_{2}(\eta) X^{10 / 6}$.

Equation (5.5) has to be solved numerically. Moreover, in view of the form of the similarity solution as $X \rightarrow 0$, it is useful to rewrite equation (5.5) in terms of $\eta=\bar{Y} / X^{1 / 3}$ and $X$, and, in order to avoid large longitudinal derivatives of the concentration near $X=0$, to introduce

$$
H \equiv\left(\phi-\phi_{s}\right) X
$$

as the dependent variable. The resulting equation is parabolic in $X$ and was discretized along then $\eta$-direction using the Galerkin finite element method with the B-cubic splines as basis functions. Integration by parts eliminates second-order derivatives and increases the accuracy of the method to $O\left(h^{3}\right)$ where $h$ is the size of the largest element. The longitudinal direction $X$ is a time-like coordinate and the integration is performed implicitly via the second-order trapezoidal rule. This gives rise to a set of nonlinear algebraic equations that are solved using Newton's iterations. Unfortunately, because $\partial \phi / \partial X$ appears inside the integrand in the second term of (5.5), the system matrix is fully populated and the CPU time increases like $N^{2}$, where $N$ is the number of unknowns. Typically 4-5 Newton's iterations were sufficient to reduce the error, defined in terms of the Euclidean norm $\left|Y^{(k+1)}-Y^{k}\right|_{2}$, to less than $10^{-8} ; Y^{k}$ denotes the $k$ th iterant of the vector of unknown coefficient of the B-spline representation of the dependent variable $H$. The computation was terminated when the particle volume fraction reached its maximum value, $\phi_{m}$, on the top face of the plate, or when it vanished at the bottom. The results presented below were checked with respect to mesh refinement and are accurate up to, at least, the third significant digit. 


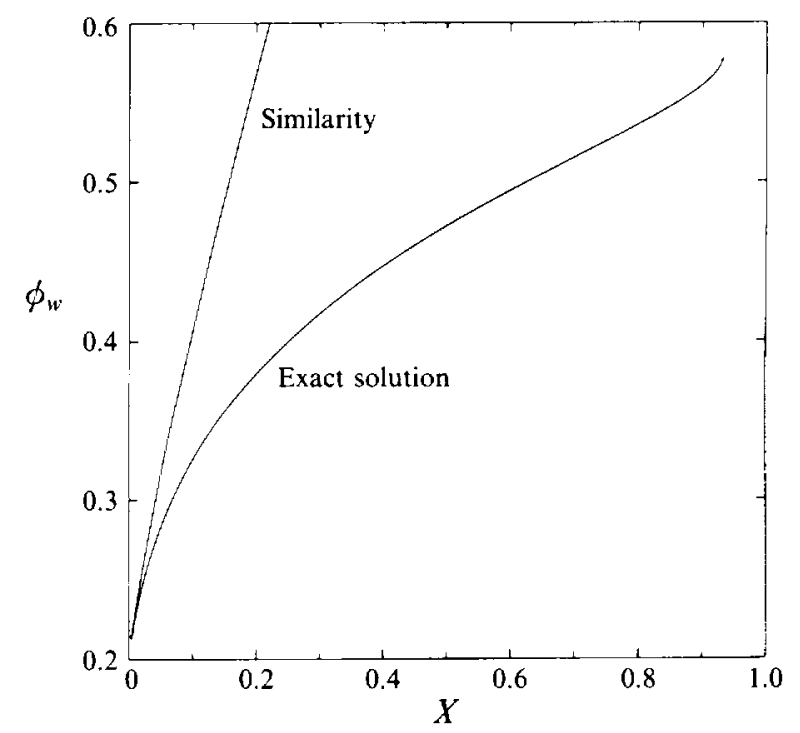

Figure 3. Comparison of the evolution of $\phi_{u}$, the particle concentration along the top face of the plate, for $\phi_{s}=0.2$, as obtained via similarity $\left(\phi_{s}+1.41 X^{5 / 6}\right)$ and as calculated via the exact solution. The computation stops when $\phi_{w}$ reaches its maximum value $\phi_{m}$.

\subsection{Results and discussion for the region above the plate}

The numerical solution of (5.5) and (5.6), to be referred to henceforth as the exact solution, generates a concentration profile, the shape of which is qualitatively similar to that given by the similarity solution. In addition, $\phi_{w}$, the particle concentration along the top side of the plate, is found to increase monotonically from the leading edge and to become equal to $\phi_{m}$ at a value of $X$ which, according to our model, is only a function of $\phi_{s}$. Beyond this point a steady-state solution of the system of equations given above does not exist and a stagnant sediment layer, having a uniform particle volume fraction $\phi_{m}$, is predicted to form whose width continuously increases in time.

The evolution of $\phi_{w}$, the concentration at the top face of the plate, both as predicted by the similarity expression, $\phi_{s}+1.41 X^{5 / 6}$, and from the exact solution, is shown in figure 3 for $\phi_{s}=0.2$. It is seen that the two results agree only up to $X \approx 0.05$, thereby implying that the range in $X$ within which the similarity solution applies is very limited. This interval can be extended by adding to the result of the similarity solution the firstorder correction, $F_{2}(\eta=0) X^{10 / 6}$, but even so, with increasing distance from the leading edge, the discrepancy between the two sets becomes rapidly more pronounced. Indeed, as shown in figure 3 , the similarity solution significantly overpredicts the rate of particle accumulation on the plate. In addition, in view of (4.1), (4.7) and (4.12), we can clearly see that the range in $X$ over which the leading-edge solution applies decreases further when $\phi_{s}$ is reduced.

On comparing the similarity solution with the exact solution two important factors are worth noting. Firstly, the shear-induced diffusion coefficient, $\beta(\phi)$, is larger than $\beta\left(\phi_{s}\right)$, which is the value used in the similarity formulation, and this reduces the rate of particle accumulation on the plate compared to that predicted by the similarity solution. On the other hand, the relative viscosity within the suspension, $\lambda(\phi)$, is larger than $\lambda\left(\phi_{s}\right)$ in the exact formulation and consequently the shear rate is smaller, which would lead to a higher rate of particle accumulation along the plate. As will be seen later, however, the effect of the increase in the shear-induced diffusion coefficient 

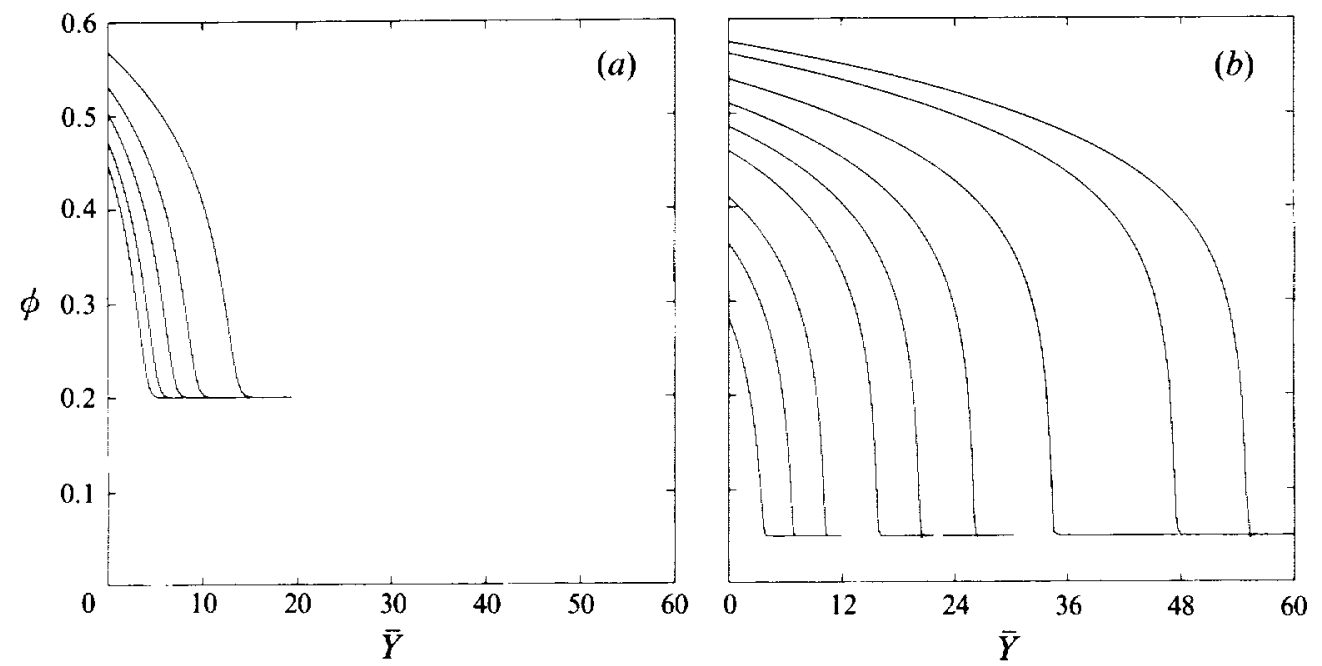

FIGURE 4. Evolution of the particle concentration profile in the region above the plate, as calculated via the exact solution at different $X$-locations downstream. $(a) \phi_{s}=0.2$ and $X=0.4,0.5,0.64,0.78$, $0.92 ; X_{c r}=0.93$ for this value of $\phi_{s}$. (b) $\phi_{s}=0.05$ and $X=0.5,1.1,1.7,2.5,3.0,3.5,4.0,4.41,4.49$; $X_{c r}=4.5$ for this value of $\phi_{s}$.

dominates particle migration near the plate, hence the exact solution leads to smaller values for $\phi_{w}$.

The above argument loses its validity as the wall concentration approaches the maximum value $\phi_{m}$, which is a singular limit in the sense that a very sharp increase in the concentration gradient, $\mathrm{d} \phi_{w} / \mathrm{d} X \rightarrow \infty$, is observed. This is a result of the rapid increase of the viscosity; $\lambda(\phi) \rightarrow \infty$ as $\phi \rightarrow \phi_{m}$. Consequently, a region of progressively smaller shear rates is formed and particles continue to accumulate on the plate as they keep sedimenting (figure 3 ). Once the concentration reaches its maximum value, a stagnant sediment layer is predicted to form which will remain attached to the plate and grow in time monotonically. In their study of sedimentation over an inclined plate, Nir \& Acrivos (1990) reached a similar conclusion when the angle of inclination fell below a certain threshold value. In the present case then, there exists a critical value of the plate length, $X_{c r}$, decreasing in magnitude with increasing $\phi_{s}$, beyond which a steady-state solution of the problem does not exist. It is understood that, in the vicinity of the region where $\phi_{w}=\phi_{m}$, the presence of the stagnant layer alters the geometry of the problem and hence the boundary-layer analysis presented in this study will no longer apply.

Figure $4(a)$ shows, for $\phi_{s}=0.2$, the evolution of the concentration profile in the region above the plate as predicted by the exact solution. The most important new feature of this exact solutions concerns the appearance of an inflection point in the concentration profile, which is seen to be moving away from the plate with increasing distance from the leading edge. The reason for this is as follows. By evaluating (5.5) at the plate we obtain

$$
\frac{\partial}{\partial \bar{Y}}\left[F(\phi)+\frac{\Delta(\phi)}{X^{1 / 2}} \frac{\partial \phi}{\partial \bar{Y}}\right]=0 \quad \text { at } \quad \bar{Y}=0
$$

which, when combined with the boundary condition (5.6), gives

$$
\frac{\partial}{\partial \bar{Y}}\left[\frac{\partial \phi}{\partial \bar{Y}}+X^{1 / 2} \frac{F}{\Delta}\right]=0 \Rightarrow \frac{\partial^{2} \phi}{\partial \bar{Y}^{2}}=X \frac{F \mathrm{~d}(F / \Delta)}{\mathrm{d} \phi} \quad \text { at } \quad \bar{Y}=0
$$


But

$$
\frac{F(\phi)}{\Delta(\phi)}=\frac{(1-\phi) \phi_{s}\left(2+\mathrm{e}^{8.8 \phi_{s}}\right)}{\left(1-\phi_{s}\right) \phi\left(2+\mathrm{e}^{8.8 \phi}\right)} \quad \text { with } \quad \phi=\phi_{w},
$$

which decreases with increasing wall concentration, owing to the rapid increase in the shear-induced diffusion coefficient with $\psi$. Consequently, $\partial^{2} \phi / \partial \bar{Y}^{2}<0$ at the plate. But since the concentration gradients are everywhere negative and become zero at the edge of the diffusion layer, the second derivative of the concentration profile must become positive at some distance from the plate, thereby explaining the existence of the inflection point.

Referring to figure $4(a)$ again, it should be noted that, with increasing $X$, the inflection point moves further away from the plate and that the thickness of the diffusion layer, i.e. the distance along the $\bar{Y}$-direction over which the particle volume fraction decreases from $\phi_{w}$, to $\phi_{s}$, increases faster with $X$ than predicted by the similarity solution. In addition, the concentration profile can be viewed as consisting of two parts. Within the first, extending between the plate and the inflection point, the concentration gradient is relatively small near the plate but increases significantly as the inflection point is approached. It will be seen later that in this region the sedimentation flux balances shear-induced resuspension, while convection remains negligibly small. Consequently, the variation of the particle volume fraction is determined primarily by the magnitude of the shear-induced diffusion coefficient. In the second region, extending from the inflection point up to the edge of the diffusion layer, the concentration gradients decay to zero exponentially fast as $\phi \rightarrow \phi_{s}$ far from the plate.

At this point it will be of interest to discuss the predictions of our model as the freestream particle volume fraction, $\phi_{s}$, varies between values close to zero and $\phi_{m}$. The evolution of the concentration profile, as computed from the exact solution for $\phi_{s}=$ 0.05 , is shown in figure $4(b)$. On comparing this figure with figure $4(a)$ which applies when $\phi_{s}=0.2$, one can see that the concentration profile remains qualitatively unaffected by the variation in $\phi_{s}$. On the other hand $X_{c r}$, the length needed for $\phi_{w}$, to equal $\phi_{m}$ on the top face of the plate, is significantly larger and the scale in the $\bar{Y}$ direction, over which changes in the concentration take place, is also larger. At the same time the concentration gradients far from the plate are larger than those observed in figure $4(a)$. In view of these two effects, and keeping in mind that the numerical discretization used in both cases was the same, the numerical error as $X \rightarrow X_{c r}$ and in the vicinity of the inflection point, where the gradients are the steepest, is very large when $\phi_{s}=0.05$. In fact, it was found that, as $\phi_{s} \rightarrow 0$ the quality of the numerical solution in this region deteriorated, for the reasons cited above, so that in order to improve the accuracy around the inflection point it would have been necessary to introduce some form of regriding. This was not pursued in this study, since the accuracy of the numerical solution near the wall, where the concentration gradients are small, was not affected. Hence, we were able to obtain reliable results for the variation of the particle volume fraction at the wall, $\phi_{w}$, for $\phi_{s}$ as small as 0.01 .

In view of the difficulties encountered in trying to compute a numerical solution as $\phi_{s} \rightarrow 0$, it seems advisable to investigate the asymptotic structure of the solution of the exact equations in this limit. Close examination of figures $4(a)$ and $4(b)$ reveals that the variation of the particle volume fraction in the transverse direction, $\bar{Y}$, is determined primarily through the balance between sedimentation and diffusion in the region near the wall. As was already mentioned in the previous paragraph but one, the concentration gradient, $\partial \phi / \partial \bar{Y}$, is small near the wall where the shear-induced diffusion 
coefficient is large, whereas far from the wall $\partial \phi / \partial \bar{Y}$ increases significantly as the particle volume fraction, and consequently the shear-induced diffusion coefficient, decreases (see also (5.11)). This so-called inner region occupies almost the entire sediment layer and determines its thickness.

In this context, and guided by the behaviour of the numerical solution for small $\phi_{s}$, we can recover, to leading order, the asymptotic structure of the exact solution as $\phi_{s} \rightarrow 0$. More specifically, in this limit, we rescale the coordinates within the inner region according to

$$
\hat{X}=X \phi_{s}^{\alpha}, \quad \hat{Y}=\bar{Y} \phi_{s}^{\beta}, \quad \alpha, \beta>0,
$$

and express the particle volume fraction, which is an $O(1)$ quantity, by means of

$$
\phi=\Phi_{0}(\hat{X}, \hat{Y})+\phi_{s} \Phi_{1}(\hat{X}, \hat{Y})+O\left(\phi_{s}^{2}\right) .
$$

We next make use of the exact integral particle balance, as obtained by integrating (5.5) across the diffusion layer and from $X=0$ to $X$,

$$
\phi_{s}^{-2 \beta} \int_{0}^{\infty}\left(\phi-\phi_{s}\right)\left(\int_{0}^{t} \frac{\mathrm{d} \rho}{\lambda(\rho)}\right) \mathrm{d} t=\phi_{s}^{-3 \alpha / 2} \hat{X}^{3 / 2}
$$

plus the fact, guided by the numerical solutions, that within the inner region the diffusion and sedimentation terms in (5.5) are of comparable magnitude and dominate those due to convection as $\phi_{8} \rightarrow 0$. Therefore, on substituting (5.12) and (5.13) into (5.14) and (5.5), we conclude that $\alpha=\frac{4}{5}, \beta=\frac{3}{5}$ and that, within the inner region $0 \leqslant$ $\hat{Y} \approx O(1)$, the particle concentration profile satisfies, as $\phi_{s} \rightarrow 0$,

$$
\frac{\partial}{\partial \hat{Y}}\left[\frac{2 \beta\left(\Phi_{0}\right)}{\hat{X}^{1 / 2} \lambda\left(\Phi_{0}\right)} \frac{\partial \Phi_{0}}{\partial \hat{Y}}+F\left(\Phi_{0}\right)\right]=O\left(\phi_{s}\right),
$$

which becomes, on integration,

$$
\frac{\partial \Phi_{0}}{\partial \hat{Y}}=-\hat{X}^{1 / 2} \frac{3\left(1-\Phi_{0}\right)}{\Phi_{0}\left(2+\mathrm{e}^{8.8 \Phi_{0}}\right)},
$$

where use has been made of (5.6), (2.7) and (2.12). The solution of (5.16) is subject to the additional constraints that

with $\delta$ given by

$$
\Phi_{0}=0 \quad \text { for } \quad \hat{Y} \geqslant \delta(\hat{X}),
$$

$$
\int_{0}^{\delta} \Phi_{0}\left(\int_{0}^{\hat{Y}} \frac{\mathrm{d} t}{\lambda(t)}\right) \mathrm{d} \hat{Y}=\hat{X}^{3 / 2},
$$

which follows from (5.14) plus (5.12) and (5.13). Note that since $\delta$ is finite, this inner solution leads to a discontinuity in the particle concentration gradient at $\hat{Y}=\delta$ which can be rendered continuous by constructing an appropriate outer solution, centred around $\hat{Y}=\delta$, to be followed by the usual matching procedure. To a first approximation, however, the existence of this outer solution does not affect the particle concentration distribution within the inner region except in the immediate neighbourhood of $\hat{Y}=\delta$.

Figure 5 shows the evolution of the particle concentration profile as given by the solution of (5.16) subject to (5.17) and (5.18). The similarity between this graph and figure $4(b)$ is obvious. The close agreement between the asymptotic solution as $\phi_{s} \rightarrow 0$ 


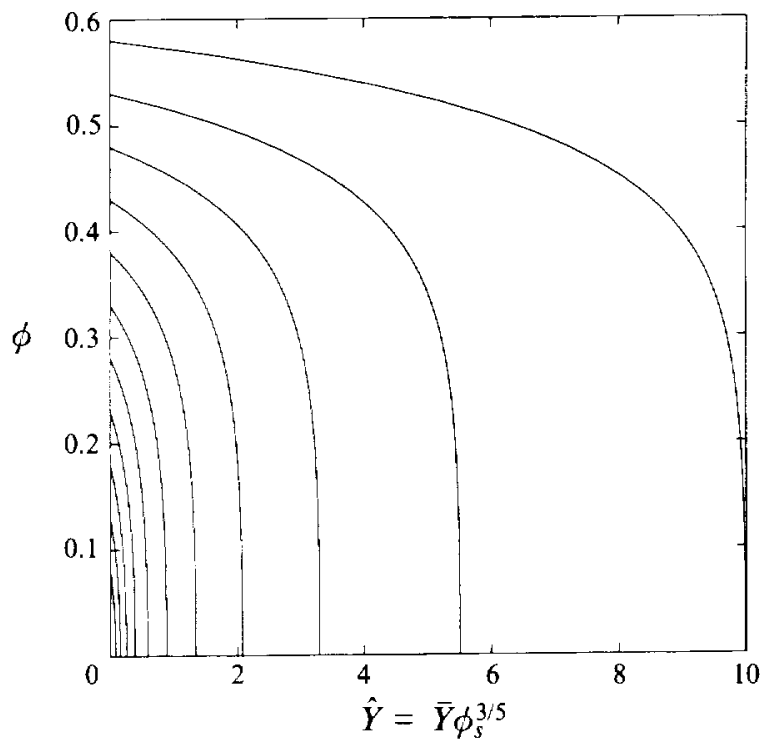

FIGURE 5. Evolution of the particle concentration profile in the region above the plate as calculated via the zeroth-order asymptotic solution as $\phi_{s} \rightarrow 0$, equations (5.17)-(5.19), at different $\hat{X}=X \phi_{s}^{4 / 5}$ locations downstream : $\hat{X}=0.0025,0.007,0.016,0.03,0.051,0.084,0.131,0.195,0.271,0.343,0.369$. $\hat{X}_{c r}=0.369$.

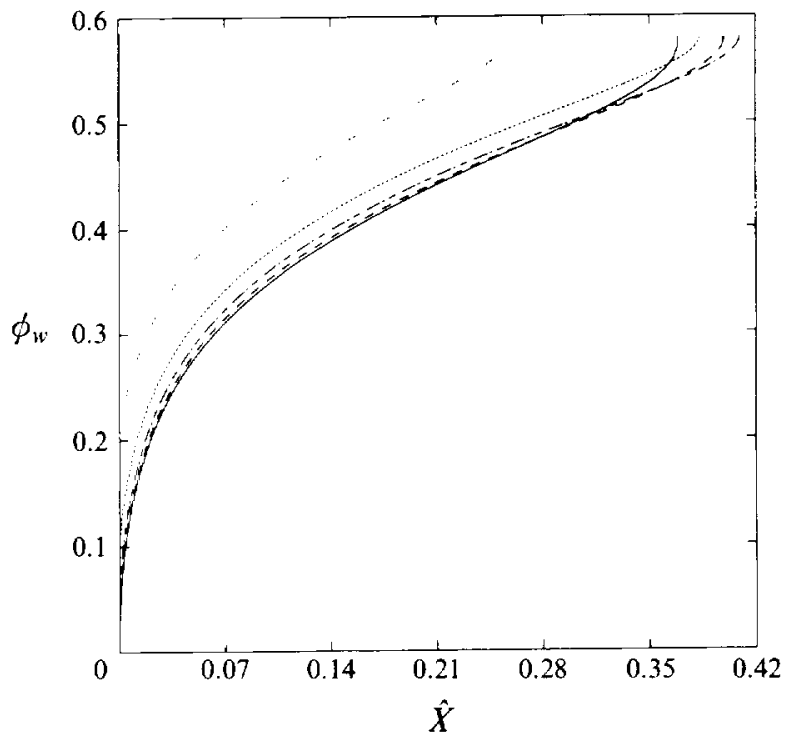

Figure 6. Comparison of the evolution of the particle concentration along the top face of the plate as obtained via the zeroth-order asymptotic solution as $\phi_{5} \rightarrow 0(-)$, and as calculated via the exact solution for different values of $\phi_{s}: \cdots \cdots \cdots, 0.2 ; \cdots, 0.1 ;-\cdot-, 0.05 ;---, 0.02$.

and the numerical solution is further illustrated in figure 6 where $\phi_{w}$, the particle volume fraction at the wall, is seen plotted $v s . \hat{X}$ for different values of $\phi_{s}$.

At the opposite extreme, $\phi_{s} \rightarrow \phi_{m}$, the structure of the solution is easier to capture. To begin within, as seen from the evolution of the particle concentration profile with 


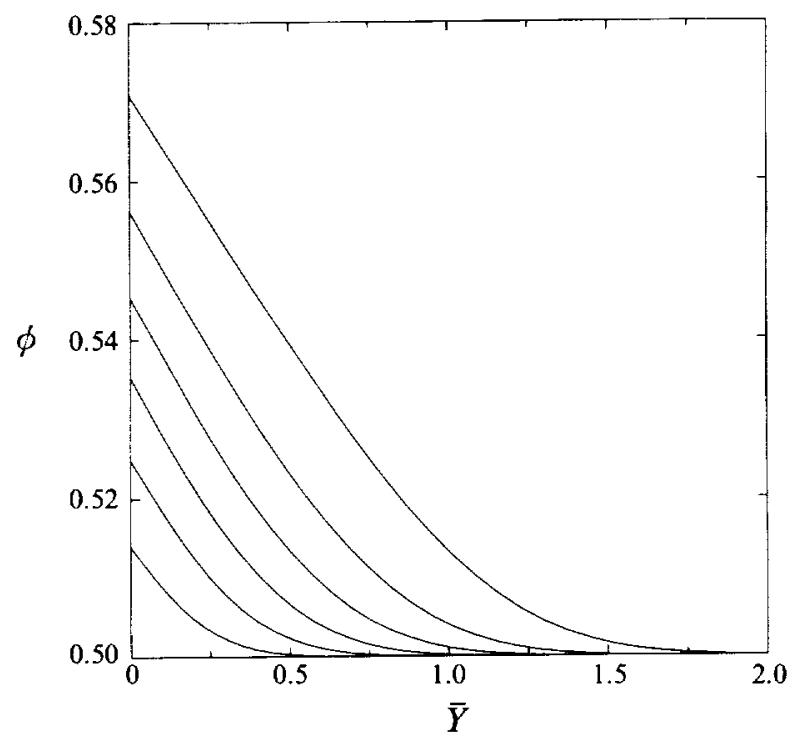

Figure 7. Evolution of the particle concentration profile in the region above the plate as calculated via the exact solution at different locations downstream : $X=0.004,0.008,0.012,0.016,0.020,0.024$ for $\phi_{s}=0.5$. $X_{c r}=0.025$ for this value of $\phi_{s}$.

$X$ when $\phi_{s}=0.5$, shown in figure 7 , the inflection point remains close to the plate even with increasing $X$. Besides, since $X_{c r}$ is now very small, the similarity solution presented in $\S 4$ applies over a larger portion of the appropriate range of $X$ than when $\phi_{s}$ was smaller. This is seen in figure 8 which depicts the variation of $\phi_{w}$ with $X$ according to the two solutions for the same value of $\phi_{s}=0.5$. Of course, $\mathrm{d} \phi_{w} / \mathrm{d} X \rightarrow \infty$ as $X \rightarrow X_{c r}$, and hence the deviations from the similarity solution become significant in this region, figure 8 . The asymptotic behaviour in this limit can be recovered by noting that

$$
\phi=\phi_{m}-\left(\phi_{m}-\phi_{s}\right) \Omega(\check{X}, \check{Y})+O\left(\phi_{m}-\phi_{s}\right)^{2} \text { as } \phi_{s} \rightarrow \phi_{m} \text {, }
$$

where

$$
\check{X}=\frac{X}{\left(\phi_{m}-\phi_{s}\right)^{\alpha}}, \quad \check{Y}=\frac{Y}{\left(\phi_{m}-\phi_{s}\right)^{\beta}}
$$

are the relevant stretched coordinates in this region. Further, by requiring that all three terms in the particle balance equation (5.5), namely convection, diffusion and sedimentation must be retained as $\phi_{s} \rightarrow \phi_{m}$, we obtain that $\alpha=\frac{6}{5}, \beta=\frac{2}{5}$, as well as the governing equation for $\Omega$ :

$$
\begin{aligned}
\frac{1}{\check{X}^{1 / 2}} \frac{\partial \Omega}{\partial \check{X}}\left(\int_{0}^{\check{Y}} \Omega^{2} \mathrm{~d} t\right)+\frac{1}{2 \check{X}^{3 / 2}} \frac{\partial \Omega}{\partial \check{Y}}\left[\int_{0}^{\check{Y}}(\check{Y}-t) \Omega^{2}\left(1+\frac{4 \check{X}}{\Omega} \frac{\partial \Omega}{\partial \check{X}}\right) \mathrm{d} t\right] \\
=\frac{\Omega^{2}}{\check{X}^{1 / 2}} \frac{\partial^{2} \Omega}{\partial \check{Y}^{2}}-\frac{2 \Omega}{\check{X}}\left(\frac{\partial \Omega}{\partial \check{Y}}\right)^{2}-2 \Omega \frac{\partial \Omega}{\partial \check{Y}},
\end{aligned}
$$

with

$$
\frac{\partial \Omega}{\partial \check{Y}}=-\check{X}^{1 / 2} \quad \text { at } \quad \check{Y}=0,
$$

and

$$
\Omega \rightarrow 1 \quad \text { as } \quad \breve{Y} \rightarrow+\infty, \quad \Omega \rightarrow 1 \quad \text { as } \quad \check{X} \rightarrow 0 .
$$




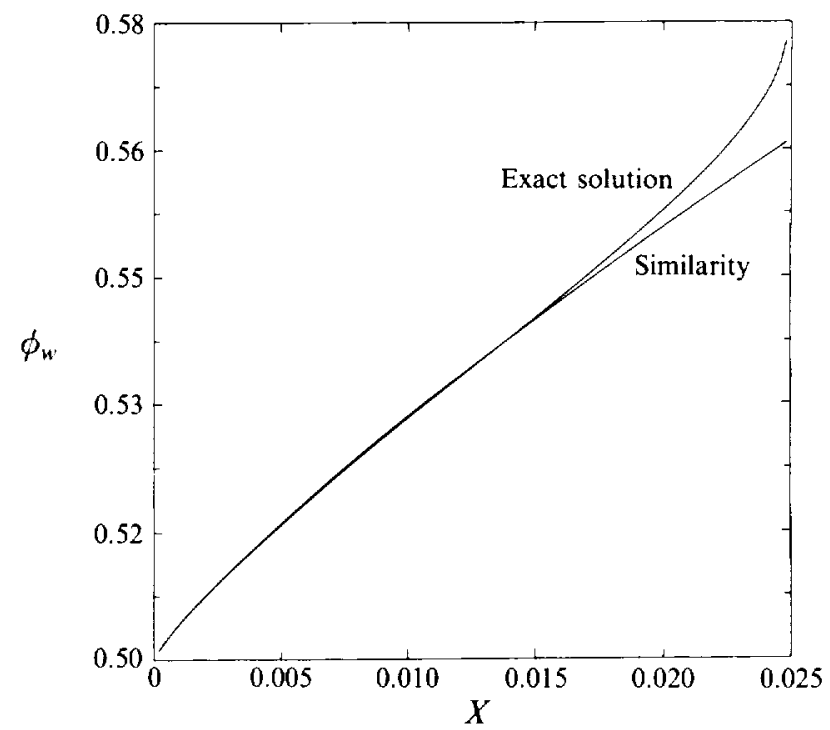

Figure 8. Comparison of the evolution of the particle concentration along the top face of the plate as obtained via similarity and as calculated via the exact equations for $\phi_{x}=0.5$.

\begin{tabular}{crrrrc}
$\phi_{s}$ & \multicolumn{1}{c}{$X_{c r}$} & $\phi_{s}$ & $X_{c r}$ & $\phi_{s}$ & $X_{r r}$ \\
0.01 & 15.000 & 0.15 & 1.485 & 0.50 & 0.0248 \\
0.02 & 9.120 & 0.20 & 0.935 & 0.53 & 0.0125 \\
0.05 & 4.497 & 0.25 & 0.580 & 0.55 & 0.0060 \\
0.08 & 3.000 & 0.30 & 0.350 & 0.57 & 0.0014 \\
0.10 & 2.415 & 0.40 & 0.114 & 0.575 & 0.0006
\end{tabular}

TABLE 1. Length along the top face of the plate needed for $\phi_{u}$ to reach its maximum possible value, $\phi_{m}=0.58$, as a function of $\phi_{s}$.

The variation of $X_{c r}$ is depicted in a $\log -\log$ scale in figure 9 while the numerically computed values of $X_{c r}$ are given in table $1 . \phi_{s} /\left(\phi_{m}-\phi_{s}\right)$ is used as the independent variable in figure 9 in order to facilitate comparison with the asymptotic results, $X_{c r} \sim 0.369 \phi_{s}^{-4 / 5}$ as $\phi_{s} \rightarrow 0$ and $X_{c r} \sim 0.352\left(\phi_{m}-\phi_{s}\right)^{6 / 5}$ as $\phi_{s} \rightarrow \phi_{m}$. The factor 0.369 was obtained by solving (5.16)-(5.18) and corresponds to the value of $\hat{X}$ for which $\Phi_{0}(\hat{Y}=0)$ becomes $\phi_{m}$, whereas the factor 0.352 corresponds to the value of $\hat{X}$ for which $\Omega(\breve{Y}=0)$ vanishes and is obtained by fitting the asymptotic result $(5.20)$ in the numerically computed values for $X_{c r}$, given in table 1 , in the limit $\phi_{s} \rightarrow \phi_{m}$. A parameter of some practical interest is the dimensional critical distance from the leading edge, $x_{c r}$, at which $\phi_{w}=\phi_{m}$. In view of (4.13), we have that

$$
\frac{x_{c r}}{a}=\left(\frac{a U_{\infty}}{v_{f}}\right)^{9 / 5}\left(\frac{v_{f}^{2} \rho_{f}}{a^{3} g \Delta \rho}\right)^{6 / 5} S
$$

where $\quad S \equiv\left(\frac{0.332\left[\beta\left(\phi_{s}\right)\right]^{2 / 3}}{\phi_{s} f\left(\phi_{s}\right)}\right)^{6 / 5}\left(1+\frac{\Delta \rho}{\rho_{f}} \phi_{s}\right)^{3 / 5}\left(1+\frac{1.5 \phi_{s}}{1-\phi_{s} / \phi_{m}}\right)^{6 / 5} X_{c r}\left(\phi_{s}\right)$,

which is a function of only $\phi_{s}$ and of the relative density ratio $\Delta \rho / \rho_{f}$. 


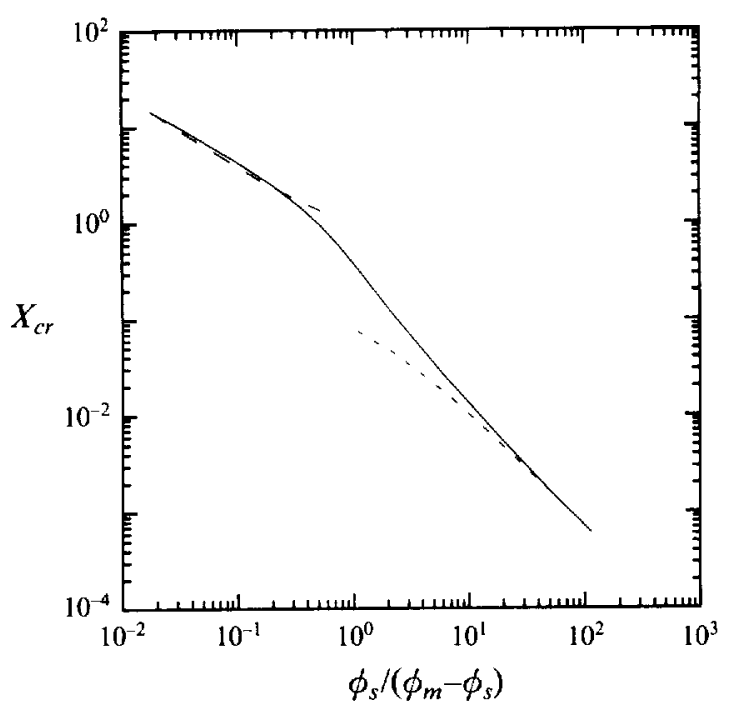

FIGURE 9. Variation, in a log-log scale, of the distance from the leading edge along the top face of the plate at which $\phi_{w}=\phi_{m}$ as a function of $\phi_{s} /\left(\phi_{m}-\phi_{s}\right)$. The continuous curve is produced by connecting the numerically obtained values whereas the two broken curves are produced by the asymptotic results as $\phi_{s} \rightarrow 0(---)$, and as $\phi_{s} \rightarrow \phi_{m}(--)$.

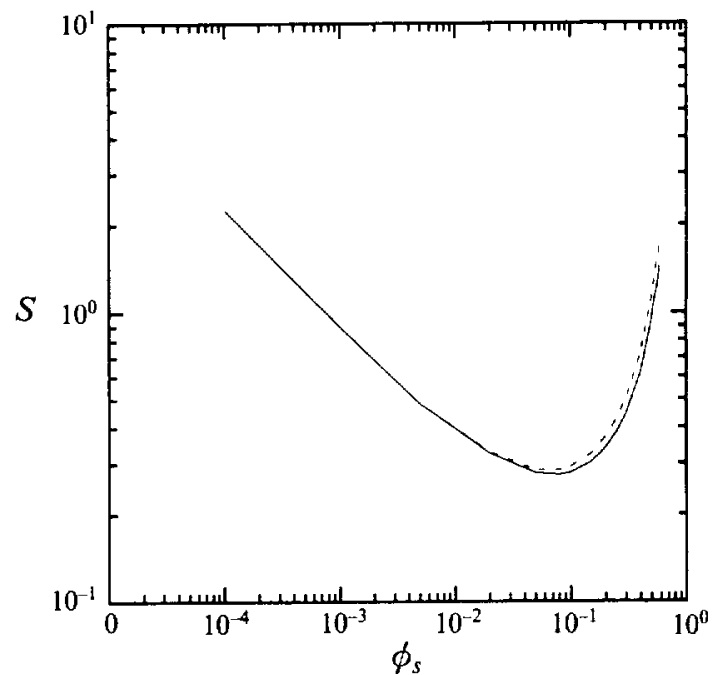

FiguRE 10. Variation of $S$, as given by (5.24), with $\phi_{s}$ for $\Delta \rho / \rho_{f}=0.2(-)$, and $1.0(---)$.

The product of the last two terms in (5.24) is a montonically decreasing function of $\phi_{s}$ and approaches a constant value when $\phi_{s} \rightarrow \phi_{m}$, as expected on account of the asymptotic behaviour of the exact equations in this limit derived above. On the other hand, since the first term in (5.24) is a rapidly increasing function of $\phi_{s}$, the complete expression for $S$ is a monotonically increasing function of $\phi_{s}$, beyond $\phi_{s} \sim 0.1$, even up to $\phi_{s}=\phi_{m}$, figure 10. This implies that when the suspension is densely packed, shear-induced diffusion is sufficiently dominant to prevent the particles from accumulating along the plate even for large distances from the leading edge. It should also be noted however that, owing to the difficulty of constructing reliable numerical 

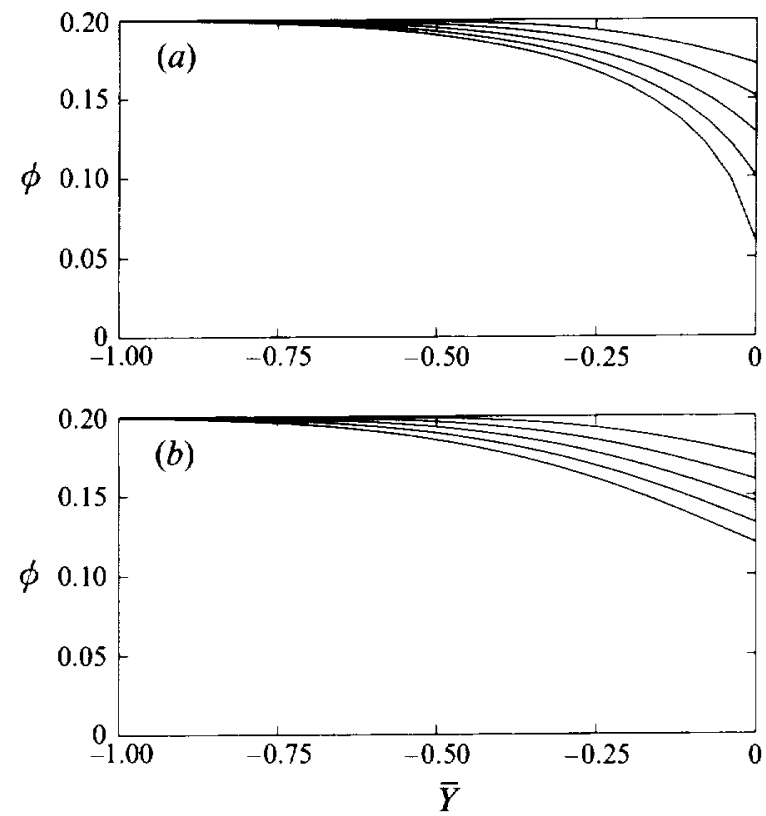

FIGURE 11. Evolution of the particle concentration profile in the region below the plate, as obtained via similarity, $(b)$, and as calculated via the exact equations, $(a)$, at different locations downstream: $X=0.008,0.014,0.02,0.026,0.032$ for $\phi_{s}=0.2 . X_{c r}=0.032$ for this value of $\phi_{g}$.

solutions for $\phi_{s}>0.575$, the curves in figure 10 were generated in this interval by multiplying the first two terms of (5.24) with the asymptotic form of the product $X_{c r} \lambda\left(\phi_{s}\right)^{6 / 5} \sim 0.352\left(\phi_{m}-\phi_{s}\right)^{6 / 5} \lambda\left(\phi_{s}\right)^{6 / 5}$. Similarly, since as was explained earlier in this section reliable numerical solutions could not be generated for $\phi_{s}$ below 0.01 , the asymptotic expression $X_{c r} \sim 0.369 \phi_{s}^{-4 / 5}$ was used in the interval $0<\phi_{s}<0.01$. The logarithmic scale used in figure 10 provides a better resolution of the curves near the endpoints of the interval $0 \leqslant \phi_{s} \leqslant \phi_{m}$. Needless to add, as $\phi_{s} \rightarrow \phi_{m}$, the variation of $S$ with $\phi_{s}$ is very sensitive to the choice of the parameter functions $\beta, f$ and $\lambda$ used in our model. In fact, since the asymptotic forms of these parameters are at present not known with any degree of confidence, the asymptotic results given above are, of course, subject to revision as more reliable expressions for $\beta, f$ and $\lambda$ become available for high particle concentrations.

\subsection{Results and discussion for the region below the plate}

Below the plate and attached to it there is a region, termed the depletion layer, where the particle concentration continuously increases from its value at the plate, $\phi_{w}$, to that in the free stream, $\phi_{s}$, owing to the continuous outflux of particles sedimenting towards the bulk of the suspension. As a result, the particle volume fraction along the underside of the plate, $\phi_{w}$, decreases from $\phi_{s}$ at the leading edge until total depletion is achieved. Not surprisingly, near the leading edge, the concentration profiles as predicted by the similarity solution and those calculated via the numerical solution of equations (5.5)-(5.6) are in very close agreement. However, as $X$ increases, the particle concentration, as obtained from the exact numerical solution, decreases rapidly with increasing distance from the wall and quickly reaches the constant value, $\phi_{s}$, at the edge of the layer, i.e. the thickness of the depletion layer is smaller than predicted by similarity; this behaviour is shown in figure 11 when $\phi_{s}=0.2$. In addition, as shown 


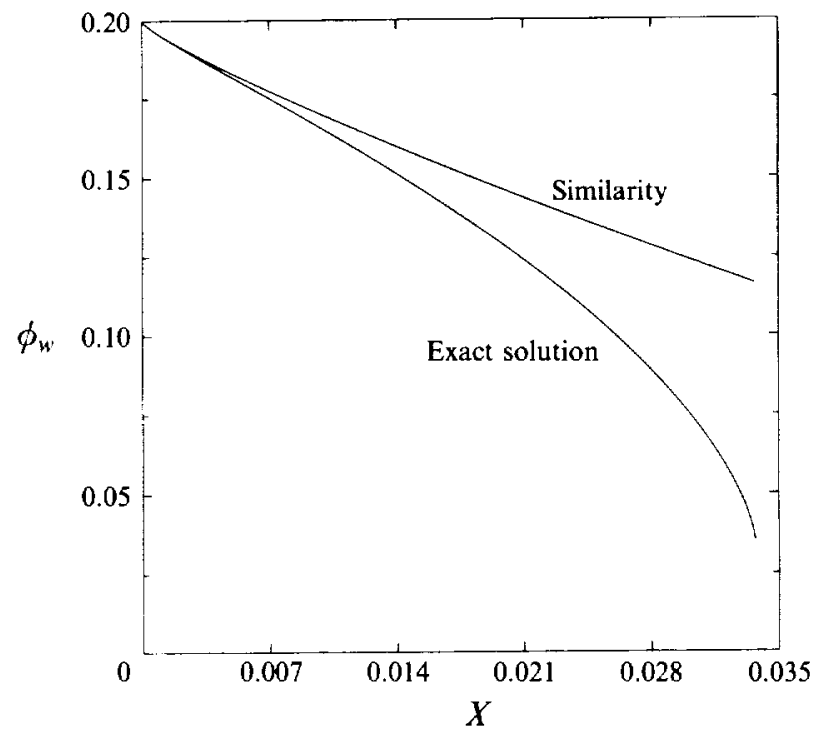

FIGURE 12. Comparison of the evolution of the particle concentration along the bottom face of the plate as obtained via similarity and as calculated via the exact equations for $\phi_{s}=0.2$.

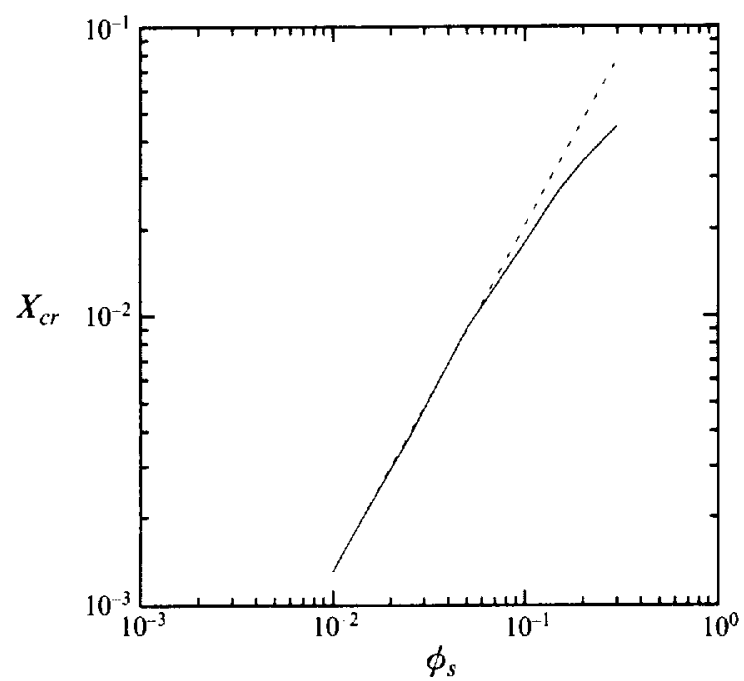

FIGURE 13. Variation, as a function of $\phi_{\varepsilon}$, of the distance from the leading edge along the bottom face of the plate at which $\phi_{x}$ first vanishes. The continuous curve is produced by connecting the numerically calculated values whereas the broken curve corresponds to the asymptotic expression as $\phi_{8} \rightarrow 0$.

in figure 12, the particle volume fraction at the wall, $\phi_{w}$, decreases with $X$ much more rapidly than predicted by the similarity solution. It should also be noted that the concentration profiles no longer have an inflection point and that, as $\phi_{w} \rightarrow 0$, very large normal gradients are observed near the wall accompanied by an unbounded growth of the longitudinal gradients; see figures 11 and 12 .

This behaviour can again be explained by an argument similar to that used earlier to account for the flow situation above the plate. Specifically, as in that case, the 


\begin{tabular}{cccc}
\hline$\phi_{s}$ & $X_{c r}$ & $\phi_{s}$ & $X_{c r}$ \\
0.0100 & 0.0013 & 0.1000 & 0.0180 \\
0.0125 & 0.0017 & 0.1500 & 0.0270 \\
0.0250 & 0.0038 & 0.2000 & 0.0340 \\
0.0500 & 0.0090 & 0.3000 & 0.0450
\end{tabular}

TABLE 2. Length along the bottom face of the plate needed for $\phi_{n}$ to vanish, as a function of $\phi_{s}$.

variation of the diffusion coefficient which is now lower than its constant value, $\beta\left(\phi_{s}\right)$, used in the similarity formulation, is the primary factor which determines the evolution of the particle volume fraction along the underside of the plate, hence the larger particle depletion rate observed in figure 12. Finally, according to (5.10), the second derivative of the particle concentration is negative along the plate, and since it remains negative throughout the layer this excludes the possibility of having an inflection point. Thus the situation is opposite to that in the region above the plate, where the inflection point moves away from the plate, and as a result the thickness of the depletion layer is smaller than predicted by similarity.

The distance from the leading edge, $X_{c r}$, at which the concentration at the wall first vanishes is plotted in figure 13 as a function of $\phi_{s}$ with the actual numerical values given in table 2. A logarithmic scale is chosen to represent the data so that the comparison with the asymptotic result as $\phi_{s} \rightarrow 0$ becomes clearer. Again an asymptotic analysis that is uniformly valid within the depletion layer can be constructed as $\phi_{s} \rightarrow 0$, and by requiring that convection should balance shear-induced diffusion and sedimentation it can easily be shown that, in this limit, an $O\left(\phi_{s}^{8 / 15}\right)$ boundary layer forms in the $\bar{Y}$-direction below the plate, that extends an $O\left(\phi_{s}^{6 / 5}\right)$ distance from the leading edge.

This research was supported in part by NASA Grant NAG 1-1159 and by the State of New York under its Einstein Chair Program.

\section{REFERENCES}

Davis, R.H. \& Birdsell, S. A. 1987 Hydrodynamic model and experiments for crossflow microfiltration. Chem. Engng Commun. 49, 217-234.

Davis, R. H. \& Leighton, D. T. 1987 Shear-induced transport of a particle layer along a porous wall. Chem. Engng Sci. 42, 275-281.

DAvis, R. H. \& SHERwoOd, J. D. 1990 A similarity solution for steady-state crossflow microfiltration. Chem. Engng Sci. 45, 3203-3209.

Dunham, R. E. 1987 The potential influence of rain on airfoil performance. Presented at the Von Karman Institute for Fluid Dynamics, Lecture Series Influence on Environmental Factors on Aircraft Wing Performance, February 1987.

KAPOOR, B. \& ACrivos, A. 1995 Flow of a sediment layer on an inclined plate. J. Fluid Mech. 290, 39-66.

Lfighton, D. \& Acrivos, A. 1986 Viscous resuspension. Chem. Engng Sci. 41, 1377-1384.

Leighton, D. \& ACrivos, A. $1987 a$ Measurement of self-diffusion in concentrated suspensions of spheres. J. Fluid Mech. 177, 109-131.

Leighton, D. \& ACrivos, A. $1987 b$ The shear-induced migration of particles in concentrated suspensions. J. Fluid Mech. 181, 415-439.

Nir, A. \& ACrivos, A. 1990 Sedimentation and sediment flow on inclined surfaces. J. Fluid Mech. $212,139-153$.

Schlichting, H. 1968 Boundary Laver Theory, 6th Edn. McGraw-Hill. 
